\section{Case report on stereotactic body radiation therapy for locally recurrent renal cell carcinoma after partial nephrectomy in a patient with single kidney}

DOI: $10.4103 /$ sajc.sajc_7_19

Dear Editor,

Patients with bilateral renal cell carcinoma (RCC) are at increased risk for the development of locally recurrent or de novo tumors after nephrectomy. ${ }^{[1]}$ When dealing with recurrent renal masses, the options are limited to observation, total nephrectomy, ablation, or repeat surgical intervention. ${ }^{[2]}$ Because surgery is not suitable for all patients, there is a need for less or noninvasive methods as treatment alternatives. ${ }^{[3]}$ Now, stereotactic body radiation therapy (SBRT) has yielded a high rate of local control for primary tumors and metastases in numerous tumor types including those of the kidneys. ${ }^{[4]}$

In this study, we present a case reporting durable control following SBRT for an inoperable local recurrence of RCC in a single partially functional kidney. This report highlights the importance of SBRT for patients with inoperable RCC.

A 52-year-old female with a 3-month history of progressive back pain, anorexia, and weight loss presented 2 years following left radical nephrectomy and partial right nephrectomy for bilateral Grade I RCC with extension to the resection margins on the right side. The pain had rapidly increased in intensity over a 2 -week period. The patient had no comorbid diseases and no other signs or symptoms.

Computed tomography (CT) scan of the abdomen showed a $3 \mathrm{~cm} \times 2 \mathrm{~cm}$ intense enhancing lesion in the mid-pole dorsal cortex of the right kidney. There was no mass in the left renal fossa. The scan was suggestive of recurrence on the right-sided partially functioning kidney. No other disease was evident on chest CT or full body bone scan [Figure 1].

The location and invasive appearance of the mass indicated local RCC recurrence from the resection margins of the previous partial right-sided nephrectomy, which was positive at that time biopsy was performed to confirm the diagnosis. CT-guided core biopsy confirmed a well-differentiated adenocarcinoma negative for CK7 and CK20 with coexpression of vimentin consistent with RCC.

South Asian Journal of Cancer Volume 8 • Issue 2 • April-June 2019
The clinical case was discussed in a multidisciplinary tumor board involving urological oncology surgeons, radiation oncologists, medical oncologists, radiologists, and pathologists. Repeat surgical intervention would have added to increased morbidity as she had only a single partially functioning kidney. Hence, in view of the isolated site of disease, it was felt that localized noninvasive treatment was preferable and SBRT was offered to the patient.

The patient underwent a treatment planning four-dimensional CT simulation scan (1-mm axial cuts) in VACLOC with body frame SBRT to assess tumor motion and assist in determining planning target volume (PTV) margins. A maximum intensity projection image set was generated and used to delineate the internal target volume (ITV). CT images were fused with simulation $\mathrm{CT}$ to assist in target delineation and dose determination. The tumor and normal critical structures were contoured by the radiation oncologist. Cortex and medulla of the remaining kidney were contoured separately as critical structures. A 2-mm margin was added on the ITV to generate the PTV. Avoidance structures include small bowel, liver, and spinal cord. Single-arc VMAT plan was used. The prescription was 3500 cGy in 5 fractions and $100 \%$ prescription dose covered $95 \%$ of the PTV. Right kidney (volume $133 \mathrm{~cm}^{3}$ ) mean dose was 1305.2 cGy. Dose to surrounding organs were within

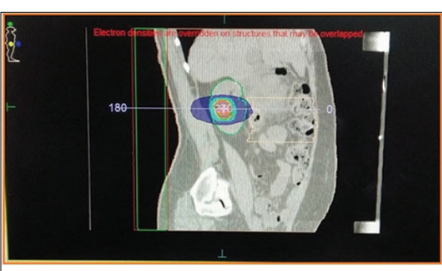

Dose distribution in a sagittal CT section

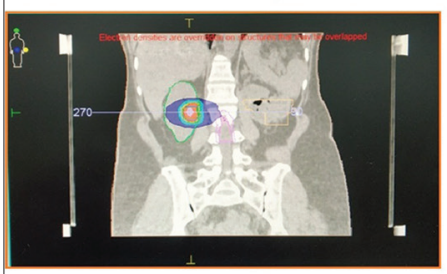

Dose distribution in a coronal CT section

Figure 1: Case report on stereotactic body radiation therapy for locally recurrent renal cell carcinoma

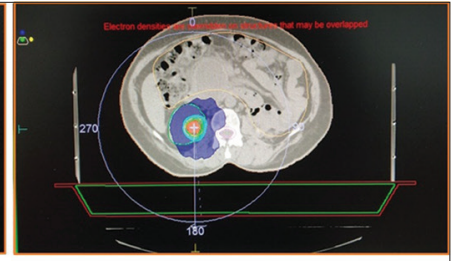

Dose distribution in a axial CT section

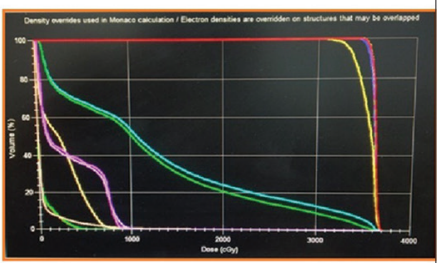

DVH paramters for target lesion and OARs 
accepted tolerance limits. The treatment plan was evaluated and showed clinically acceptable dose distributions with conformity index of the prescription isodose to the PTV of 0.08. Image guidance was used for all fractions. The patient was treated on alternate days.

Within $24 \mathrm{~h}$ of the first fraction of SBRT, the patient experienced an initial pain flare requiring an increase in analgesia. However, the pain had completely resolved in 1 week following treatment allowing discontinuation of all analgesics. She returned to regular physical activity within 6 weeks. She had no other acute toxicities related to the SBRT. Magnetic resonance imaging (MRI) of the abdomen 4 months posttreatment showed a slight reduction in the dimensions of the mass, but a significant change in signal characteristics with central tumor necrosis indicating treatment response. Her renal functions had also returned to baseline values. Her case was rediscussed at the multidisciplinary tumor board, and a surveillance approach with 4 monthly MRIs was planned. MRI 9 months post-SBRT showed a reduction in the dimensions of the mass to $1 \mathrm{~cm} \times 0.7 \mathrm{~cm}$. Clinically, the patient remained asymptomatic and active. No biopsy was performed, and the multidisciplinary team recommended continued surveillance of the patient. 15 months post-SBRT treatment with regular follow-up, she is doing well with normal renal functions. Last MRI 11 months posttreatment was suggestive of a radiologically stable disease with no further increase in the size of the lesion. Serial renal function tests which include serum creatinine and blood urea also remained within normal limits.

In summary, we have described how SBRT can achieve durable control of a locally recurrent $\mathrm{RCC}$, a tumor type traditionally thought to be radioresistant. This emphasizes the need to reevaluate the role of therapies with advances in technology and underlines the benefit of multidisciplinary collaboration in challenging cases.

\section{Declaration of patient consent}

The authors certify that they have obtained all appropriate patient consent forms. In the form the patient(s) has/have given his/her/ their consent for his/her/their images and other clinical information to be reported in the journal. The patients understand that their names and initials will not be published and due efforts will be made to conceal their identity, but anonymity cannot be guaranteed.

Financial support and sponsorship

Nil.

\section{Conflicts of interest}

There are no conflicts of interest.

Kaushik Jagannath Kataki, Ajinkya Gupte, Ram Madhavan, K. Beena, Debnarayan Dutta, Raghavendra Holla', Manoj Kalita ${ }^{2}$

Departments of Radiation Oncology and 'Radiation Physics, Amrita Institute of Medical Sciences, Kochi, Kerala, ${ }^{2}$ Department of Biostatistics,

Dr. B. Borooah Cancer Institute, Guwahati, Assam, India Correspondence to: Mr. Manoj Kalita, E-mail: manojkalita5354@gmail.com

\section{References}

1. Bratslavsky G, Linehan WM. Long-term management of bilateral, multifocal, recurrent renal carcinoma. Nat Rev Urol 2010;7:267-75.

2. Acar Ö, Şanlı Ö. Surgical management of local recurrences of renal cell carcinoma. Surg Res Pract 2016;2016:2394942.

3. Bhat S. Role of surgery in advanced/metastatic renal cell carcinoma. Indian J Urol 2010;26:167-76.

4. Blomgren H, Lax I, Näslund I, Svanström R. Stereotactic high dose fraction radiation therapy of extracranial tumors using an accelerator. Clinical experience of the first thirty-one patients. Acta Oncol 1995;34:861-70.

This is an open access journal, and articles are distributed under the terms of the Creative Commons Attribution-NonCommercial-ShareAlike 4.0 License, which allows others to remix, tweak, and build upon the work non-commercially, as long as appropriate credit is given and the new creations are licensed under the identical terms.

How to cite this article: Kataki KJ, Gupte A, Madhavan R, Beena K, Dutta D, Holla $\mathrm{R}$, et al. Case report on stereotactic body radiation therapy for locally recurrent renal cell carcinoma after partial nephrectomy in a patient with single kidney. South Asian J Cancer 2019;8: 135-6.

(c) 2019 The South Asian Journal of Cancer | Published by Wolters Kluwer - Medknow 